\title{
Meandering instability of a rivulet
}

\author{
By HO-YOUNG KIM ${ }^{1}$, JIN-HO KIM ${ }^{1}$ \\ AND BYUNG HA KANG ${ }^{2}$ \\ ${ }^{1}$ Thermal/Flow Control Research Center, Korea Institute of Science and Technology, \\ Seoul 136-791, Korea \\ ${ }^{2}$ Department of Mechanical and Automotive Engineering, Kookmin University, Seoul 136-702, Korea
}

(Received 3 September 2002 and in revised form 25 August 2003)

A rivulet is a narrow stream of liquid flowing down a solid surface. When its flow rate exceeds a certain limit, it tends to meander, exhibiting instability of the interface. Here we report a perturbation analysis of this meandering rivulet. We find that the combined effects of the tangential velocity difference across the interface and the dynamic wetting are responsible for the instability. The Weber number represents the ratio of the destabilizing force, inertia, to the stabilizing force, surface tension. As the Weber number increases, both the wavenumber of maximum instability and the cutoff wavenumber increase. The effects of the capillarity are such that the wavenumber of maximum instability asymptotically increases as the sensitivity of the dynamic contact angles to the contact line speed increases. However, the cutoff wavenumber remains constant despite wetting parameter changes when the Weber number is constant.

\section{Introduction}

When a liquid is supplied on a highly hydrophilic solid surface, a film flow arises. On a relatively non-wetting surface, however, a narrow liquid stream, i.e. a rivulet, is formed. Examples of this rivulet flow include a water stream on a windowpane in a rainy day as well as liquid streams used in evaporation heat exchangers, absorption refrigerators and cooling towers. The rivulet exhibits diverse flow patterns as Kern $(1969,1971)$ and Schmuki \& Laso (1990) observed. That is, as the flow rate increases, the flow develops in the sequence droplet flow, linear straight rivulet, meandering rivulet and turbulent oscillating rivulet.

A static rivulet is a liquid thread without an axial velocity component in its base state and its instability was first addressed by Davis (1980). Schiaffino \& Sonin (1997) simplified the analysis by assuming inviscid quasi-one-dimensional liquid flow. They found their approximate modelling results to agree fairly well with experimental measurements using molten wax beads of characteristic length less than $100 \mu \mathrm{m}$, even with the inviscid approximation. For a dynamic rivulet having an axial velocity component in its base state, an axisymmetric instability causing it to break up into drops was analysed by Young \& Davis (1987). In the analysis, a fully developed base flow approximation was employed. They found that the rivulet break-up mechanism is similar to the capillary instability of a liquid jet, except for an effect of the dynamic contact line, in that a high pressure in a pinched region pushes liquid into a thicker region. However, their analysis, based on the lubrication flow approximation, only predicts a varicose instability, failing to predict a sinuous or meandering instability.

In addition to the aforementioned studies on rivulets, the behaviour of viscous liquid jets under a compressive force was studied by Taylor (1969) and Tchavdarov, 
Yarin \& Radev (1993). Buckling of those jets appears similar to meandering of rivulets. However, the buckling phenomenon is regarded as irrelevant to the rivulet meandering for the following reasons. The meandering disappears as the flow velocity decreases when viscosity is more likely to dominate. It is also well known that the meandering becomes extremely pronounced as the flow rate increases when the effect of viscosity diminishes. For very viscous liquids, only a linear rivulet occurs when the liquid is supplied onto an inclined surface (Schmuki \& Laso).

The goal of the present work is to investigate the instability mechanism that disturbs a straight rivulet into a meander. Here we note that the rivulet's meandering instability is amplified as the flow rate increases as the foregoing rivulet flow regime studies suggest. This implies that the inertial component plays an important role in destabilizing a rivulet. It is also natural to relate the meandering instability to the Kelvin-Helmholtz instability which arises when the layers of different fluids are in relative tangential motion (Chandrasekhar 1961). In this context, the instability of liquid streams surrounded by an ambient gas was studied by Hagerty \& Shea (1955), Debye \& Daen (1959) and Yang (1992). Hagerty \& Shea studied the instability of a liquid sheet ejected into air and showed that the sinuous instability indeed takes place. Debye \& Daen extended the analysis to sheets and cylinders with surface or body tension. Yang investigated the instability of a cylindrical liquid jet taking into account the effects of the ambient gas. His perturbation analysis revealed that when the inertia increases as compared with the surface tension, an asymmetric mode including a sinuous perturbation is excited. However, those flow configurations studied above are different from a rivulet, which is in contact with a solid surface and thus involves dynamic contact lines. The character of the change of the dynamic contact angles with the contact line speed determines the boundary condition of the flow and also flow instability. Regarding the mechanism behind rivulet meandering, Culkin \& Davis (1984) suggested that inertia and surface tension destabilize and stabilize the rivulet, respectively, under the effects of dynamic wetting. Hence a stability index, i.e. a criterion for meandering, was given by the ratio of inertia to surface tension.

In this work, we perform a perturbation analysis to study the rivulet's sinuous or meandering instability, which has long been observed experimentally but not yet theoretically clarified. We investigate the effects of the wetting properties including equilibrium and dynamic contact angles and of the flow rate on the rivulet instability. In addition, comparisons are made between our theoretical predictions and measurement results both previously reported and obtained by an experiment performed in this work.

\section{Formulation}

Consider a rivulet flowing down a solid surface with the width $2 a$ as shown in figure 1. The liquid is assumed to be inviscid and the validity of this is discussed in the next section. The flow is taken to be quasi-two-dimensional, assuming a negligible $z$-direction velocity compared with the velocities parallel to the solid plane and consequently a negligible $z$-direction pressure gradient. Then the governing equation is the Laplace equation of the velocity potential $\phi$ :

$$
\frac{\partial^{2} \phi}{\partial x^{2}}+\frac{\partial^{2} \phi}{\partial y^{2}}=0 .
$$

It is supposed that the velocity potential and the flow boundary are slightly disturbed from a basic state in which a straight rivulet flows with the $x$-direction velocity $U$. 
(a)

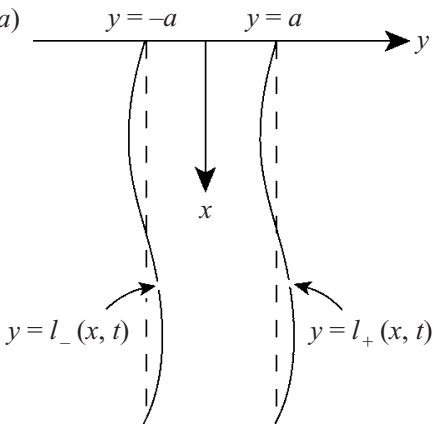

(b)

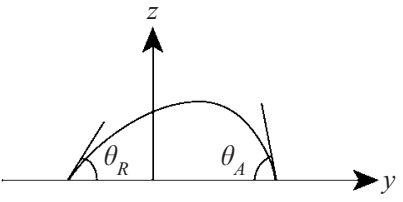

FIgURE 1. Schematic of an undulated rivulet. (a) Top view; $(b)$ cross-sectional view.

Then the velocity potential of the liquid, $\phi_{l}$, is written as a sum of the base solution $\phi_{l}^{(0)}$ and the first-order perturbation $\phi_{l}^{(1)}$ :

$$
\begin{aligned}
\phi_{l} & =\phi_{l}^{(0)}+\phi_{l}^{(1)} \\
& =U x+(A \cosh k y+B \sinh k y) \exp (\mathrm{i} k x+\mathrm{i} \omega t), \quad-a \leqslant y \leqslant a,
\end{aligned}
$$

where $k$ and $\omega$ denote the wavenumber and angular frequency of the disturbance, respectively. The velocity potentials of the gas on either side of the rivulet are

$$
\begin{aligned}
\phi_{g+} & =\phi_{g+}^{(0)}+\phi_{g+}^{(1)} \\
& =U^{\prime} x+G_{+} \exp (-k y+\mathrm{i} k x+\mathrm{i} \omega t), \quad y>a,
\end{aligned}
$$

and

$$
\begin{aligned}
\phi_{g-} & =\phi_{g-}^{(0)}+\phi_{g-}^{(1)} \\
& =U^{\prime} x+G_{-} \exp (k y+\mathrm{i} k x+\mathrm{i} \omega t), \quad y<-a .
\end{aligned}
$$

The kinematic boundary conditions at $y= \pm a$ are such that

$$
\frac{\mathrm{D}}{\mathrm{D} t}\left(y-l_{+}\right)=0, \quad y=a,
$$

and

$$
\frac{\mathrm{D}}{\mathrm{D} t}\left(y-l_{-}\right)=0, \quad y=-a,
$$

where the rivulet edge perturbations, $l_{+}$and $l_{-}$, are written as

$$
l_{+}=L \exp (\mathrm{i} k x+\mathrm{i} \omega t)
$$

and

$$
l_{-}=L \exp (\mathrm{i} k x+\mathrm{i} \omega t+\mathrm{i} \theta) .
$$

The first-order expressions of (2.5) and (2.6) become

$$
\frac{\partial \phi^{(1)}}{\partial y}=\frac{\partial l_{ \pm}}{\partial t}+\frac{\partial \phi^{(0)}}{\partial x} \frac{\partial l_{ \pm}}{\partial x}, \quad y= \pm a,
$$

for both liquid and gas. Then we can express $A, B, G_{+}$and $G_{-}$in terms of $L$ as

$$
A=\frac{\mathrm{i}((\omega / k)+U)\left(1-\mathrm{e}^{\mathrm{i} \theta}\right)}{2 \sinh k a} L,
$$




$$
\begin{aligned}
& B=\frac{\mathrm{i}((\omega / k)+U)\left(1+\mathrm{e}^{\mathrm{i} \theta}\right)}{2 \cosh k a} L, \\
& G_{+}=-\mathrm{i}\left((\omega / k)+U^{\prime}\right) \mathrm{e}^{k a} L, \\
& G_{-}=\mathrm{i}\left((\omega / k)+U^{\prime}\right) \mathrm{e}^{\mathrm{i} \theta+k a} L .
\end{aligned}
$$

The zeroth-order dynamic boundary condition (DBC) at $y=a$ is

$$
\frac{1}{2}\left(\rho_{l} U^{2}-\rho_{g} U^{\prime 2}\right)+\frac{\sigma}{R_{b}}=\Delta P_{0}
$$

where $\rho_{l}$ and $\rho_{g}$ are the densities of the liquid and gas, respectively, $\sigma$ is the liquid's surface tension, $R_{b}^{-1}$ the curvature of the rivulet cross-section in the base state, and $\Delta P_{0}$ the pressure adjustment. The first-order DBC at $y=a$ is

$$
\rho_{l} \frac{\partial \phi_{l}^{(1)}}{\partial t}-\rho_{g} \frac{\partial \phi_{g+}^{(1)}}{\partial t}+\rho_{l} \nabla \phi_{l}^{(0)} \cdot \nabla \phi_{l}^{(1)}-\rho_{g} \nabla \phi_{g+}^{(0)} \cdot \nabla \phi_{g+}^{(1)}+\sigma\left(-\frac{\partial^{2} l_{+}}{\partial x^{2}}+\delta \kappa_{+}\right)=0,
$$

where $\delta \kappa_{+}$denotes the disturbance of rivulet curvature in the $(y, z)$-plane. This curvature disturbance is related to the dynamic contact angle, i.e. the deviation of the contact angle from the equilibrium value due to the motion of the contact line. We emphasize that although the rivulet curvature arises both in the $(x, y)$ - and $(y, z)$ planes, the dominant capillary pressure difference develops in the $y$-direction. This is consistent with the two-dimensional modelling employed here. The dynamic contact angle is a function of the contact line speed in general (Dussan V. 1979). Here we assume a linear relationship between the curvature disturbance and the contact line speed $u_{C L}$ :

$$
\delta \kappa_{ \pm}=\frac{C}{R_{b}} u_{C L, \pm}
$$

where the subscript \pm denotes the value at $y= \pm a$. As shown in Appendix $\mathrm{A}, u_{C L}$ is given by

$$
u_{C L, \pm}=\frac{\partial l_{ \pm}}{\partial t} .
$$

The coefficient $C$ in (2.16) can be related to the dynamic and equilibrium contact angles as shown in Appendix B. The first-order DBC at $y=-a$ is

$$
\rho_{l} \frac{\partial \phi_{l}^{(1)}}{\partial t}-\rho_{g} \frac{\partial \phi_{g-}^{(1)}}{\partial t}+\rho_{l} \nabla \phi_{l}^{(0)} \cdot \nabla \phi_{l}^{(1)}-\rho_{g} \nabla \phi_{g-}^{(0)} \cdot \nabla \phi_{g-}^{(1)}+\sigma\left(\frac{\partial^{2} l_{-}}{\partial x^{2}}-\delta \kappa_{-}\right)=0 .
$$

After substituting (2.10), (2.11) and (2.12) into (2.15), and also (2.10), (2.11) and (2.13) into (2.18), we rearrange the two algebraic equations to give

$$
\left[\rho_{l} k\left(\frac{\omega}{k}+U\right)^{2} \operatorname{coth} k a+\rho_{g} k\left(\frac{\omega}{k}+U^{\prime}\right)^{2}-\sigma\left(k^{2}+\mathrm{i} \frac{C}{R_{b}} \omega\right)\right]\left(1-\mathrm{e}^{\mathrm{i} \theta}\right)=0
$$

and

$$
\left[\rho_{l} k\left(\frac{\omega}{k}+U\right)^{2} \tanh k a+\rho_{g} k\left(\frac{\omega}{k}+U^{\prime}\right)^{2}-\sigma\left(k^{2}+\mathrm{i} \frac{C}{R_{b}} \omega\right)\right]\left(1+\mathrm{e}^{\mathrm{i} \theta}\right)=0 .
$$

The simultaneous equations (2.19) and (2.20) are satisfied either when $\theta=0$ and

$$
\rho_{l} k\left(\frac{\omega}{k}+U\right)^{2} \tanh k a+\rho_{g} k\left(\frac{\omega}{k}+U^{\prime}\right)^{2}-\sigma\left(k^{2}+\mathrm{i} \frac{C}{R_{b}} \omega\right)=0
$$


or when $\theta=\pi$ and

$$
\rho_{l} k\left(\frac{\omega}{k}+U\right)^{2} \operatorname{coth} k a+\rho_{g} k\left(\frac{\omega}{k}+U^{\prime}\right)^{2}-\sigma\left(k^{2}+\mathrm{i} \frac{C}{R_{b}} \omega\right)=0 .
$$

The former case corresponds to the sinuous perturbation and the latter to the varicose perturbation.

We note that when $C$ is set to be zero, i.e. when the variation of the curvature in a plane perpendicular to the solid surface is neglected, (2.21) and (2.22) become identical to the results of Hagerty \& Shea and Debye \& Daen. This is when the problem is strictly two-dimensional. When $C$ is not zero, the foregoing quasi-twodimensional governing equation still neglects the $z$-direction contribution. This in turn makes the boundary conditions involving $z$-coordinates mathematically unnecessary. Consideration of the pressure distribution in the quasi-two-dimensional picture leads to the assumption that the meandering in the $(x, y)$-plane is due to the pressure gradient in the $(x, y)$-plane, which makes a good physical sense. Our dynamic boundary conditions impose only two pressure jump conditions, corresponding respectively to advancing and receding fronts. This is consistent with the geometric approximation that the rivulet cross-section is made up of two arcs having radii $R_{A}$ and $R_{R}$ as explained in Appendix B.

We rearrange (2.21) to examine instability by the sinuous perturbations:

$$
(\eta+\tanh k a) \omega^{2}+k\left(2 U \tanh k a-\mathrm{i} \frac{\sigma}{\rho_{l}} \frac{C}{R_{b}}\right) \omega+k^{2} U^{2} \tanh k a-\frac{\sigma}{\rho_{l}} k^{3}=0,
$$

where $\eta$ denotes the ratio of the gas density to the liquid density. Equation (2.23) is non-dimensionalized with the time scale taken to be $\tau=a / U$ :

$$
(\eta+\tanh \tilde{k}) \tilde{\omega}^{2}+\tilde{k}\left(2 \tanh \tilde{k}-\mathrm{i} \frac{\beta}{W e} \tilde{C}\right) \tilde{\omega}+\tilde{k}^{2} \tanh \tilde{k}-\frac{\tilde{k}^{3}}{W e}=0
$$

where $\tilde{\omega}=a \omega / U, \tilde{k}=k a, \tilde{C}=C U, \beta=a / R_{b}$, and the Weber number $W e=\rho_{l} U^{2} a / \sigma$. It is noted that $\beta$ is determined solely by the equilibrium contact angle $\theta_{e}$ when the gravitational effect is negligible. In such a case, $\beta=\sin \theta_{e}$. The rivulet is unstable to the sinuous perturbations when $\tilde{\omega}$ includes imaginary parts. We employ computer software to solve (2.24) and to examine the perturbation growth rate.

\section{Results and discussion}

As indicated by (2.24), the response of a rivulet to sinuous perturbations is dependent on the density ratio $\eta$, the Weber number and the wetting-related properties, such as $\theta_{e}$ and $\tilde{C}$. Since (2.24) contains complex coefficients, we resort to a numerical procedure to investigate the character of rivulet instability. Here we consider a water rivulet flowing in air down a surface of Parafilm M laboratory sealing film (American National Can, Chicago, IL) as a control condition, which corresponds to our experiment described towards the end of this section. In this case, $\eta=0.00121$ and $\theta_{e}=97.4^{\circ}$.

Figure 2(a) shows the growth rate of perturbations corresponding to the dimensionless wavenumber $\tilde{k}$ for different Weber numbers. Here the growth rate $q$ denotes the negative imaginary part of $\tilde{\omega}$ having a greater magnitude than the other imaginary part. As the Weber number increases while the other conditions remain unchanged, both the wavenumber of the maximum instability and cutoff wavenumber 

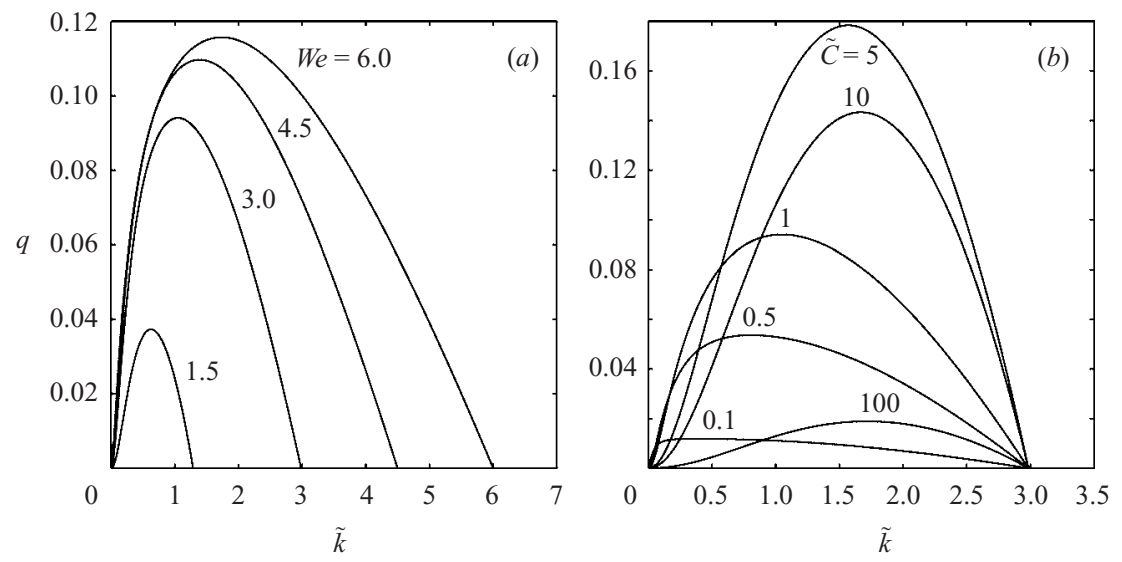

FIGURE 2. Growth rate versus $\tilde{k}$ with $\eta=0.00121$ and $\theta_{e}=97.4^{\circ}$. (a) The Weber number is varied with a constant $\tilde{C}=1 ;(b) \tilde{C}$ is varied with a constant $W e=3$.
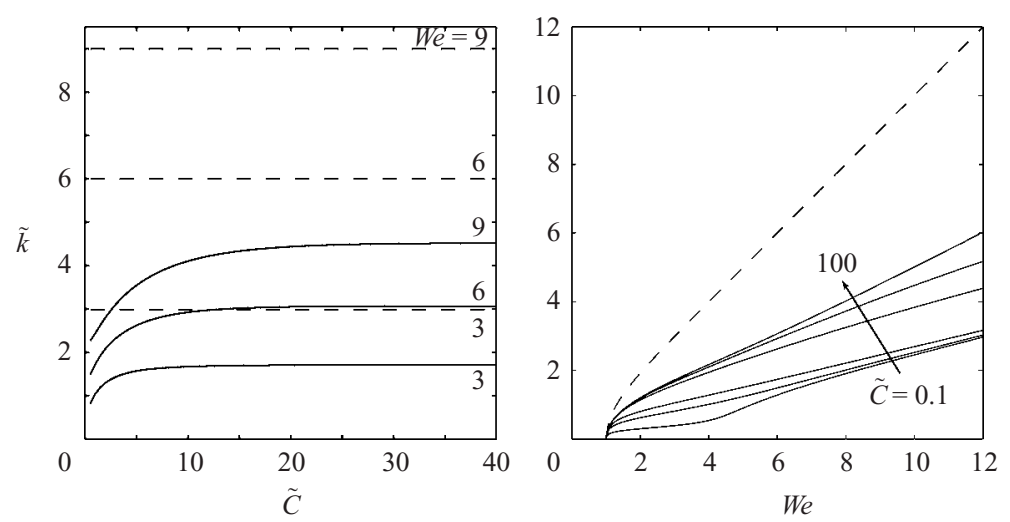

Figure 3. Dependence of the wavenumber of maximum instability (solid line) and the cutoff wavenumber (broken line) on $(a) \tilde{C}$ and $(b) W e$. In $(b), \tilde{C}=0.1,0.5,1,5,10$ and 100.

increase. Figure $2(b)$ shows the characteristics of instability as $\tilde{C}$, the dynamic wetting parameter, varies while $W e$ is kept constant. The wavenumber of maximum instability increases as $\tilde{C}$ increases but the growth rate tends to decrease after initially increasing with the increase of $\tilde{C}$.

Figure 3 summarizes the dependence of the wavenumber of maximum instability $\tilde{k}_{\max }$ and the cutoff wavenumber on $W e$ and $\tilde{C}$. It is clear that as $W e$ increases, $\tilde{k}_{\max }$ increases. In more physical terms, the most dangerous wavelength decreases as the rivulet inertia becomes stronger compared with the surface tension. Figure 3(a) shows that $\tilde{k}_{\max }$ increases with $\tilde{C}$ when $\tilde{C}$ is small but the rate of increase starts to saturate as $\tilde{C}$ exceeds about 10 . We recall that $\tilde{C}$ represents how sensitively the curvature of the rivulet cross-section, or dynamic contact angle, changes with the contact line speed. This tendency therefore indicates that $\tilde{k}_{\max }$ is proportional to the sensitivity of the dynamic contact angle to the contact line speed when the sensitivity is low. However, when $\tilde{C}$ is fairly large, which limit leads to the contact angle hysteresis, $\tilde{k}_{\max }$ varies little. The figure also reveals that the cutoff wavenumber shows no change with varying $\tilde{C}$ at a given Weber number. We note that the dynamic wetting effect, or non-zero 
$\tilde{C}$, dramatically increases both $\tilde{k}_{\max }$ and the cutoff wavenumber compared with those wavenumbers corresponding to $\tilde{C}=0$. When $\tilde{C}=0$, we obtain $\tilde{k}_{\max }=0.0016,0.0036$ and 0.0056 for $W e=3,6$ and 9 respectively. Figure $3(b)$ directly reveals such an influence of $W e$ on $\tilde{k}_{\max }$ : that $\tilde{k}_{\max }$ is proportional to $W e$ at a given $\tilde{C}$. On the other hand, we find that the cutoff wavenumbers for different $\tilde{C}$ collapse to a single line, which is consistent with the observation made in figure $3(a)$. Figure $3(b)$ also suggests that the rivulet is stable, regardless of $\tilde{C}$, for $W e<1$. It is difficult to derive this condition from the general case (2.24). However, in limiting cases where the coefficient of $\tilde{\omega}$ is either purely real or imaginary, the critical We determining the rivulet stability can be easily obtained. When $2 \tanh \tilde{k} \gg \beta \tilde{C} / W e$, the following condition should be satisfied for sinuous perturbations to grow:

$$
f(\tilde{k})=(\eta+\tanh \tilde{k}) \frac{\tilde{k}^{3}}{W e}-\eta \tilde{k}^{2} \tanh \tilde{k}<0 .
$$

Since we are interested in the behaviour of $f(\tilde{k})$ near the critical Weber number where $\tilde{k} \ll 1$, we approximate $\tanh \tilde{k} \approx \tilde{k}$ and (3.1) reduces to

$$
\tilde{k}<\eta(W e-1) .
$$

Therefore, a rivulet is always stable when $W e<1$. In the other limiting case where $2 \tanh \tilde{k} \ll \beta \tilde{C} / W e, \tilde{\omega}$ is found to be

$$
\tilde{\omega}=\frac{1}{2 A}\left[\mathrm{i} B \pm\left(-B^{2}-4 A D\right)^{1 / 2}\right],
$$

where $A=\eta+\tanh \tilde{k}, B=\tilde{k} \beta \tilde{C} / W e$, and $D=\tilde{k}^{2} \tanh \tilde{k}-\tilde{k}^{3} / W e$. For the perturbations to grow, $\tilde{\omega}$ should have a negative imaginary part and thus it follows that

$$
\frac{\tilde{k}}{\tanh \tilde{k}}<W e .
$$

Since the minimum value that $\tilde{k} / \tanh \tilde{k}$ can have is 1 , the critical Weber number is again found to be 1 .

Figure 4 reconfirms the observation from figure $2(b)$ that the maximum growth rate, $q_{\max }$, at a given condition initially increases with the increase of $\tilde{C}$ but drops after reaching a maximum. Therefore, we may note that there exists a $\tilde{C}$ value, or a dynamic wetting condition, which makes the rivulet most unstable for a given Weber number. In addition, those $\tilde{C}$ values corresponding to the maximum of $q_{\max }$ increase as $W e$ increases.

Figure 5 shows the effect of the density ratio on $\tilde{k}_{\max }$. There exists a density ratio which minimizes $\tilde{k}_{\max }$ for a given condition and it decreases as $W e$ increases. Furthermore, $\tilde{k}_{\max }$ varies more sensitively to a change of $\eta$ as $W e$ increases.

Among those factors affecting the rivulet instability, the effect of $\beta$ has not yet been investigated. In fact, $\beta$, or alternatively $\theta_{e}$, cannot independently vary without causing a change of $\tilde{C}$. That is, once we alter the liquid/solid combination, both the static and dynamic wetting properties change. The dependence of the rivulet instability characteristics on $\beta$ can be inferred with ease by observing the influence of $\tilde{C}$.

To verify our theoretical predictions, we perform a simple experiment and measure the wavelength of a meandering rivulet. In the experiment, water is supplied through a $1.6 \mathrm{~mm}$ diameter nozzle by a syringe pump. The solid surface is parafilm as mentioned at the start of this section and is inclined $10^{\circ}$ with respect to the horizontal. When the flow rate is too low, a droplet flow emerges as Schmuki \& Laso observed, but when it 


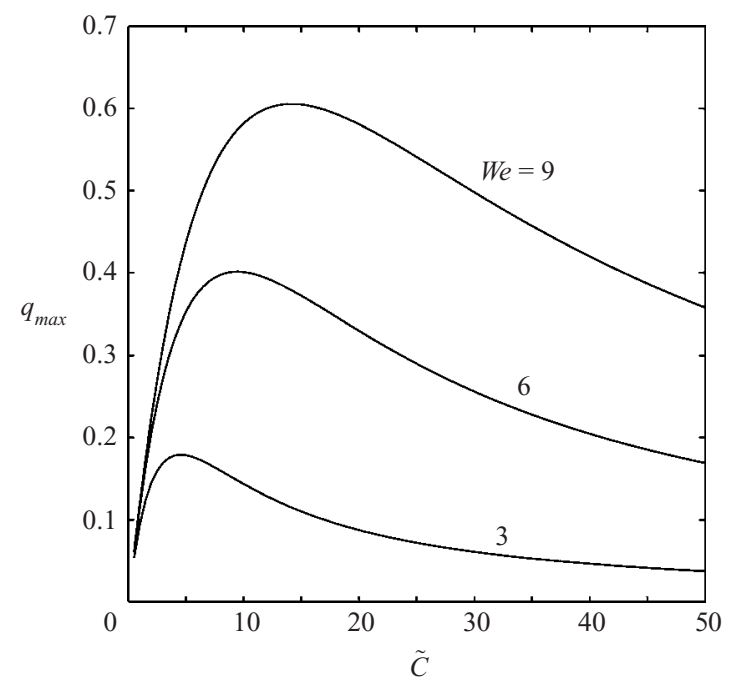

FIGURE 4. Maximum growth rate at $\tilde{k}_{\max }$ versus $\tilde{C}$ for varying $W e$.

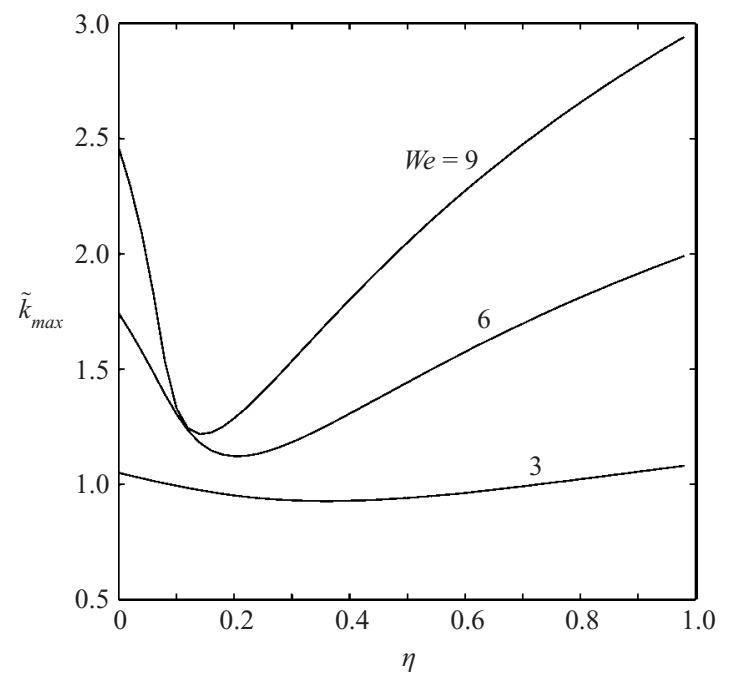

FIGURE 5. $\tilde{k}_{\max }$ versus $\eta$ for varying $W e$ with a constant $\tilde{C}=1$.

is too high, the rivulet tends to accelerate due to gravity. A typical experimental result, when the rivulet acceleration is insignificant, is a meandering wavelength of about $14 \mathrm{~mm}$ for rivulet width and the flow rate of $2.7 \mathrm{~mm}$ and $0.93 \mathrm{~cm}^{3} \mathrm{~s}^{-1}$, respectively. The average axial flow velocity is $0.27 \mathrm{~m} \mathrm{~s}^{-1}$. An experimental image of the rivulet is shown in figure 6 . This is indeed a reasonable value as we frequently observe in our daily lives including those of water traces on bathtubs and dishwashers.

Comparison of the experimental measurement with the theoretical prediction requires knowledge of the dynamic wetting properties, as represented by $C$, in dimensional form, in this work. Evaluation of $C$ involves the measurement of dynamic contact angles and contact line speed as discussed in Appendix B. We measured those quantities using a water drop sliding on an inclined parafilm surface (Kim, Lee $\&$ Kang 2002), which revealed that $C$ is approximately 3.5 and thus $\tilde{C} \approx 1$ for the 


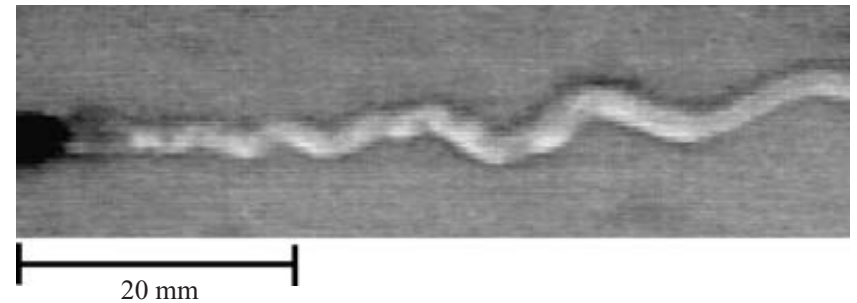

FIGURE 6. Photograph of a water rivulet flowing down a parafilm surface. Water flows from left to right.

measured values of $u_{1}=18 \mathrm{~mm} \mathrm{~s}^{-1}, \theta_{A}=102^{\circ}$ and $\theta_{R}=94^{\circ}$. Here $u_{1}$ is the magnitude of both the advancing (positive value) and receding (negative value) contact line speed since the sliding drop has both advancing and receding edges. Our theory predicts that when $W e=1.4$ and $C=3.5$, the most dangerous meandering wavelength is $14.5 \mathrm{~mm}$, which agrees well with the experimental result. In addition to our own experimental result, we compare our theoretical prediction with the experimental results reported by Nakagawa \& Scott (1984). In their experiments, a water rivulet on Plexiglas was employed with the flow rate of $1.72 \mathrm{~cm}^{3} \mathrm{~s}^{-1}$ and the corresponding $W e=1.1$. The sliding drop method results in $u_{1}=28 \mathrm{~mm} \mathrm{~s}^{-1}, \theta_{A}=104^{\circ}$ and $\theta_{R}=22^{\circ}$. This gives $C=3.9$ with $\theta_{e}=62^{\circ}$ and the most dangerous meandering wavelength is calculated to be $82 \mathrm{~mm}$. This value agrees with the rivulet wavelength shown in figure 5 of Nakagawa \& Scott for waves within $150 \mathrm{~mm}$ of the nozzle.

Here we address the question of whether our inviscid modelling is applicable to the foregoing experimental conditions. For an inviscid instability assumption to be valid, the ratio of the time scale for the fastest-growing instability to develop to the momentum diffusion time scale should be much smaller than unity (Schiaffino \& Sonin):

$$
\frac{v}{q^{*} a^{2}} \ll 1,
$$

where $v$ is the kinematic viscosity of the liquid and $q^{*}$ the dimensional growth rate at $\tilde{k}_{\max }$. This ratio is calculated to be 0.076 and 0.36 for the current experiment and that of Nakagawa \& Scott, respectively. Therefore, our inviscid modelling is more appropriate for the current experiment. Another criterion for the viscous effects not to affect most of the rivulet core is given by

$$
X \ll h^{2} U / \nu,
$$

where $X$ is the distance from the nozzle and $h$ is the rivulet height. We have $h \approx 1.4 \mathrm{~mm}$ for the current experiment because the contact angle is close to $90^{\circ}$. Then (3.6) gives $X \ll 600 \mathrm{~mm}$ and it is applicable to the current experiment. In the case of Nakagawa \& Scott, $h^{2} U / v$ is $660 \mathrm{~mm}$ and thus the region considered for the comparison above satisfies (3.6).

\section{Concluding remarks}

Although the varicose instability of a rivulet contacting solid and the asymmetric instability of a jet in air have previously been analysed, the rivulet meandering instability has not been theoretically addressed thus far. Therefore, we have analysed, for the first time, such a meandering instability of a rivulet in this work. We conclude with a discussion of the nature of this instability in the following. 
(a)

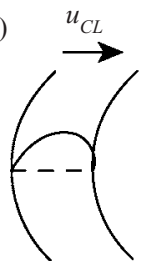

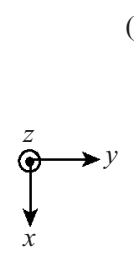

(b)

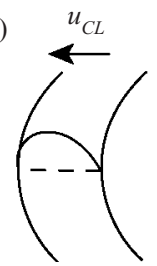

FIGURE 7. Effect of capillary forces on instability. The curvature in the $(x, y)$-plane induces higher liquid pressure on the left both in $(a)$ and $(b)$ and thus stabilizes the disturbance, i.e. it tends to straighten the rivulet. On the other hand, the curvature in the $(y, z)$-plane as shown in $(a)$ is such that the higher liquid pressure is developed on the right. Hence it promotes the meandering undulation. The curvature in the $(y, z)$-plane of $(b)$ develops higher liquid pressure on the left and thus stabilizes the flow.

The fundamental mechanism behind this instability is in part related to the tangential velocity difference at the liquid/gas interface. At the same time, the capillary force due to the curvature in the $(x, y)$-plane (figure 1) always stabilizes sinuous disturbances as it tends to straighten the rivulet. The Weber number represents the ratio of the destabilizing force (inertia) to the stabilizing force (surface tension). Our theory predicts that as $W e$ increases, both the wavenumber of maximum instability and the cutoff wavenumber increase. On the other hand, the dynamic wetting effects, i.e. the curvature variation at moving contact lines, are rather subtle. The effect of the capillary force related to the contact line movement depends on the flow direction. Therefore, as shown in figure 7, it may either destabilize or stabilize the flow, independent of the perturbed rivulet shape. We presume that the cutoff wavenumber is kept constant at a given Weber number, as in figure 3(a), due to such effects of the dynamic wetting.

\section{Appendix A. Contact line speed}

The contact line speed, $u_{C L}$, corresponds to the rate of motion of the contact line normal to itself, and is written as

$$
u_{C L}=d_{x} u_{x}+d_{y} u_{y},
$$

where $d_{x}$ and $d_{y}$ denote the direction-cosines of the normal at $(x, y)$ and $u_{x}$ and $u_{y}$ are the velocities in the $x$-and $y$-directions, respectively. When the contact line is described as $F(x, y, t)=y-l(x, t)=0$, we have (Lamb 1932)

$$
d_{x}=\frac{\partial F / \partial x}{\xi} \quad \text { and } \quad d_{y}=\frac{\partial F / \partial y}{\xi},
$$

where $\xi=\left[(\partial F / \partial x)^{2}+(\partial F / \partial y)^{2}\right]^{1 / 2}$. It is easy to show that $\xi \approx 1$ thus $d_{x} \approx-\partial l / \partial x$ and $d_{y} \approx 1$. Therefore (A 1$)$ becomes

$$
u_{C L}=-\frac{\partial l}{\partial x} u_{x}+u_{y}
$$

Combining (A 2) with the kinematic boundary condition leads to

$$
u_{C L}=\frac{\partial l}{\partial t} .
$$




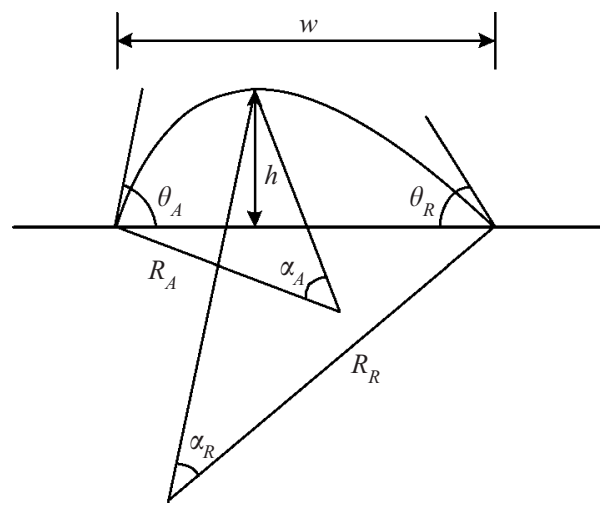

Figure 8. Geometry for a cross-section of a dynamic rivulet.

\section{Appendix B. Curvature and dynamic contact angles}

Here we show that the coefficient, $C$, appearing in (2.16) can be expressed in terms of $\theta_{e}$, the advancing contact angle $\theta_{A}$ and the receding contact angle $\theta_{R}$ when a few assumptions are made. As shown in figure 8 , we assume that the maximum height of a rivulet lies where the arcs having radii $R_{A}$ and $R_{R}$ meet and that the liquid partially wets the solid $\left(0 \ll \theta_{e} \ll \pi\right)$. Then we write

$$
\alpha_{j}=2\left\{\theta_{j}-\tan ^{-1}\left[\frac{h}{w}\left(\cot \theta_{A}+\cot \theta_{R}\right) \tan \theta_{j}\right]\right\},
$$

where $j$ denotes $A$ or $R$ and $w$ the rivulet width. Geometric considerations give $R_{A}$ and $R_{R}$ as

$$
R_{j}=\frac{\cot \theta_{j}\left[w^{2}+h^{2}\left(\cot \theta_{A}+\cot \theta_{R}\right)^{2} \tan ^{2} \theta_{j}\right]^{1 / 2}}{\sqrt{2}\left(1-\cos \alpha_{j}\right)^{1 / 2}\left(\cot \theta_{A}+\cot \theta_{R}\right)} .
$$

When $\alpha_{j} \ll 2 \theta_{j}$ and the distortion of the rivulet due to dynamic wetting is not severe, i.e. $2 h / w \approx \tan \left(\theta_{e} / 2\right)$, a tedious algebraic procedure leads to

$$
\frac{1}{R_{j}}=\frac{\sin \left(\theta_{j}-\theta_{e} / 2\right)}{\sqrt{2} R_{b}\left(1-\cos \theta_{e}\right)^{1 / 2}} .
$$

If a linear relationship between the dynamic contact angle and the contact line speed is assumed (Greenspan 1978), we have

$$
\theta_{j}=\frac{\theta_{j, 1}-\theta_{e}}{u_{1}} u_{C L}+\theta_{e},
$$

where $\theta_{j, 1}$ denotes a dynamic contact angle corresponding to a contact line velocity $u_{1}$. We assume that there is no contact angle hysteresis. It can be readily shown that this linear relationship is similar to Hoffman's law (Hoffman 1975) written as

$$
u_{C L}=\gamma\left(\theta_{A}^{3}-\theta_{e}^{3}\right),
$$

where $\gamma$ is the empirical constant (Fermigier \& Jenffer 1991), when $\left(\theta_{A}-\theta_{e}\right) / \theta_{e} \ll 1$. Based on the foregoing assumptions, we approximate (B 4$)$ as follows:

$$
\frac{\sin \left(\theta_{j}-\theta_{e} / 2\right)}{\sin \left(\theta_{e} / 2\right)}=C^{*} u_{C L}+1
$$


where $C^{*}$ is given by

$$
C^{*}=\frac{\sin \left[\left(\theta_{A, 1}-\theta_{R, 1}\right) / 2\right]}{u_{1} \tan \left(\theta_{e} / 2\right)} .
$$

It is interesting to note that (B 3) can be rewritten as

$$
\frac{1}{R}=\frac{1}{R_{b}}+\frac{C^{*}}{R_{b}} u_{C L},
$$

where the advancing contact line corresponds to positive $u_{C L}$ and the receding contact line to negative $u_{C L}$. Then we find that (2.16) and (B 8) are equivalent and thus $C=C^{*}$.

\section{REFERENCES}

Chandrasekhar, S. 1961 Hydrodynamic and Hydromagnetic Stability. Clarendon. (Also Dover, 1981.)

Culkin, J. B. \& Davis, S. H. 1984 Meandering of water rivulets. AIChE J. 30, 263-267.

Davis, S. H. 1980 Moving contact lines and rivulet instabilities. Part 1. The static rivulet. J. Fluid Mech. 98, 225-242.

Debye, P. \& Daen, J. 1959 Stability considerations on nonviscous jets exhibiting surface or body tension. Phys. Fluids 2, 416-421.

Dussan V., E. B. 1979 On the spreading of liquids on solid surfaces: static and dynamic contact lines. Annu. Rev. Fluid Mech. 11, 371-400.

Fermigier, M. \& JenfFer, P. 1991 An experimental investigation of the dynamic contact angle in liquid-liquid systems J. Colloid Interface Sci. 146, 226-241.

Greenspan, H. P. 1978 On the motion of a small viscous droplet that wets a surface. J. Fluid Mech. 84, 125-143.

Hagerty, W. W. \& Shea, J. F. 1955 A study of the stability of plane fluid sheets. J. Appl. Mech. 22, $509-515$.

Hoffman, R. L. 1975 A study of the advancing interface. I. Interface shape in liquid-gas systems. J. Colloid Interface Sci. 50, 228-241.

KeRn, J. 1969 Zur Hydrodynamik der Rinnsale. Verfarenstechnik 3, 425-430.

KERN, J. 1971 Stabilitätsprobleme der Rinnsalströmung. Verfarenstechnik 5, 289-294.

Kim, H.-Y., LeE, H. J. \& Kang, B. H. 2002 Sliding of liquid drops down an inclined solid surface. J. Colloid Interface Sci. 247, 372-380.

Lamb, H. 1932 Hydrodynamics, 6th Edn. Cambridge University Press.

NaKagawa, T. \& ScotT, J. C. 1984 Stream meanders on a smooth hydrophobic surface. J. Fluid Mech. 149, 89-99.

Schiaffino, S. \& Sonin, A. A. 1997 Formation and stability of liquid and molten beads on a solid surface. J. Fluid Mech. 343, 95-110.

Schmuki, P. \& Laso, M. 1990 On the stability of rivulet flow. J. Fluid Mech. 215, 125-143.

TAYLOR, G. I. 1969 Instability of jets, threads, and sheets of viscous fluid. Proc. 12th Intl Congr. Appl. Mech., Stanford, 1968 (ed. M. Hetényi \& W. G. Vincenti), p. 382. Springer.

Tchavdarov, B., Yarin, A. L. \& Radev, S. 1993 Buckling of thin liquid jets. J. Fluid Mech. 253, 593-615.

Yang, H. Q. 1992 Asymmetric instability of a liquid jet. Phys. Fluids A 4, 681-689.

Young, G. W. \& Davis, S. H. 1987 Rivulet instabilities. J. Fluid Mech. 176, 1-31. 